\title{
EL TURISMO GASTRONÓMICO EN LIMA METROPOLITANA: DIAGNÓSTICO DE LA OFERTA Y LA DEMANDA
}

\author{
GASTRONOMIC TOURISM IN LIMA METROPOLITAN: SUPPLY AND DEMAND DIAGNOSIS
}

David Carreño Farfán* Ivdad007@hotmail.com

[RECEPCIÓN: MARZO DE 2015 / CONFORMIDAD: ABRIL DE 2015]

RESUMEN

Este proyecto de investigación se centra en el turismo gastronómico de Lima Metropolitana, localizado dentro del denominado "centro histórico", a través de la identificación de las empresas de alimentos y bebidas que constituyen la oferta existente frente al comportamiento del turista y sus exigencias, con la finalidad de elaborar un diagnóstico de la situación y plantear alternativas para su desarrollo y mayor satisfacción de ambas partes. El trabajo se plantea como un estudio que puede dar la pauta a otros estudios relacionados con este tema e incluso en otros espacios geográficos, ya que la tendencia del sector gastronómico en el Perú continúa creciendo de manera sostenible. Asimismo, se pretende brindar un aporte que contribuya al desarrollo de este tipo de turismo poniendo los resultados obtenidos en conocimiento de los representantes del sector privado, como la Sociedad Peruana de Gastronomía (APEGA), y los gobiernos locales.

Palabras clave: Gastronomía, turismo, capacitación, oferta, demanda, pequeña empresa.

\begin{abstract}
This research project focuses on the Gastronomic Tourism of Lima. It is located within so-called "Historical Center" through the identification of Food\&Beverage companies constituting the existing supply against tourist behavior and requirements. It aims to develop a situational analysis and to propose alternatives for their development and greater satisfaction of both parties. The work is proposed to be a study that can guide other surveys related to this subject and studies in other geographic areas, as the trend of the gastronomic industry in Peru issustain ably growing. It also aims to provide a contribution to help develop this type of tourism giving knowledge to the private sector as well as the Peruvian Society of Gastronomy and local government through Councils and responsible sectors for the area.
\end{abstract}

Keywords: Gastronomy, tourism, training; supply, demand, small business.

* Magíster en Educación (IPLAC de Cuba), Licenciado en Historia (UNMSM), Técnico en Cocina (Le Cordón Bleu-Perú). Docente Auxiliar de la Facultad de Ciencias Administrativas de la Universidad Nacional Mayor de San Marcos en el área de Gastronomía. 


\section{INTRODUCCIÓN}

El turismo, como actividad económico-productiva, ha venido creciendo en los últimos años de manera acelerada en el país. Las estimaciones dadas a conocer para este año expresan que "... el turismo receptivo del Perú crecería 6\% en 2015, aseveró el Gremio de Turismo de la Cámara de Comercio de Lima (CCL). Esta tendencia se verá impulsada por el mayor ingreso de turistas provenientes de Chile y de Estados Unidos" (Perú 21 2015). Su presencia repartida a lo largo del territorio permite impulsar muchos sectores que sostienen dicha actividad. El incremento en cifras reales del turismo ya contabilizadas para el 2014, así como la identificación de su procedencia, permite ver con optimismo las posibilidades de crecimiento, además de establecer sus particularidades y las características de sus demandas.

Según el reporte de la Comisión de Promoción del Perú para la Exportación y el Turismo (PROMPERÚ 2014), en su publicación "Perfil del Turista Extranjero 2013”, el Perú recibió a 3.16 millones de turistas internacionales, quienes durante su estadía gastaron un total de US\$ 3,925 millones. Por su parte, la Cámara de Comercio de Lima (CCL) señala que el año pasado "el ingreso de turistas chilenos y estadounidenses lideró el mercado en nuestro país durante 2014. Según la Superintendencia Nacional de Migraciones, Perú recibió a 1'446,689 visitantes chilenos y 312,086 estadounidenses"(Peru21 2015). Es decir, se conoce cuál es el grupo más representativo que visita la ciudad y de acuerdo a ello se puede identificar mejor sus particularidades y costumbres.

A partir de ello el trabajo se centra en la ciudad capital y por eso es necesario conocer el número de turistas que visitan Lima y cuáles son sus características más saltantes Es así que PROMPERÚ ha dado información muy valiosa al respecto (El Comercio 2014) señalando, por ejemplo, que el $72 \%$ de los turistas que vienen al país visitan particularmente Lima y su promedio de permanencia es de seis días, en los cuales el $35 \%$ se hospeda en hoteles de 4 o 5 estrellas, otro $35 \%$ en hoteles de 3 estrellas, un $23 \%$ en hoteles de 1 o 2 estrellas y el restante $22 \%$, en casas de amigos o familiares. El informe también nos ilustra que en el 2013 Lima recibió $62 \%$ de turistas hombres y $38 \%$ mujeres y la procedencia más significativa era principalmente de Estados Unidos (20\%), Chile (9\%), Argentina (6\%), Brasil (6\%) y Colombia (6\%).
Para ir especificando el campo de acción de la presente investigación, se ha establecido que de todo ese conglomerado de visitantes, el lugar más visitado en nuestra capital es el centro de Lima -con un 77\%- y Larcomar -con 10\%-. Los distritos donde más van los turistas son Miraflores, San Isidro y Barranco. La gran mayoría de visitantes llega a hacer turismo cultural (87\%); luego, un $40 \%$ realiza turismo de naturaleza, un $24 \%$ turismo de aventura y un pequeño $11 \%$ viene a visitar la playa.

De acuerdo a ello, se puede estimar que para el año 2013 (del cual ya se tienen cifras oficiales) los turistas extranjeros que han visitado el centro de Lima -con mayor precisión el denominado "centro histórico"- bordean el 1'751,904 personas. Esto genera de por sí un conjunto de necesidades de servicios básicos que debe ser cubiertos de la mejor manera. Dentro de estos, se pueden mencionar servicios tan simples y elementales como traductores, guías, oficinas de informes, casas de cambio, servicios de seguridad, baños y, sobre todo, satisfacer la demanda de alimentación, haciéndola plácida y accesible para todos los bolsillos del turista. Lo peor que puede pasar es que visite el centro y se sirva sus alimentos en otro lugar.

Se especifica en la presente investigación que el centro histórico de Lima ha sido declarado Patrimonio de la Humanidad por la UNESCO por su gran variedad de atractivos de riqueza histórica, numerosos conjuntos monumentales de los siglos XVII y XVIII, arquitectura civil y religiosa con una variedad de estilos, barrios tradicionales, galerías artesanales y restaurantes.

\section{PLANTEAMIENTO DEL PROBLEMA}

De acuerdo a lo descrito en la introducción, el tema de la propuesta conjuga los requerimientos de los turistas, principalmente a través de su alimentación y la oferta de ella por parte de los diferentes locales de expendio de alimentos y bebidas (A\&B) situados en el centro histórico, en lo que hoy conocemos con el "turismo gastronómico". Trabajos previos alrededor del tema se han desarrollado principalmente en artículos periodísticos, en su mayoría sin brindar un consolidado serio sobre el boom gastronómico vigente. Carecemos todavía de un estudio que nos brinde un panorama completo de la cadena gastronómica, mostrando sus articulaciones con los diversos sectores de la economía nacional e internacional. El aporte económico y social de la gastronomía en 
el Perú, de Arellano Marketing (2009), realizado por encargo de la Sociedad Peruana de Gastronomía (APEGA), constituye uno de los trabajos más serios realizados hasta la actualidad, dándonos una visión macro del panorama gastronómico. Lo que se busca en la presente investigación es precisar el tema, situándose con mayor propiedad en la zona de Lima metropolitana.

Desde hace aproximadamente 10 años el país viene experimentado un pujante desarrollo productivo vinculado al sector alimentario, el cual busca -entre otras cosas- reconocer el trabajo de la tierra y el mar a través de la preservación de ingentes recursos provenientes de muchas zonas del país. El desarrollo económico ha hecho posible esto. La preservación y producción de insumos, así como su puesta en valor, se debe también a factores objetivos (muy particulares del país) como la geografía y el clima, que son elementos determinantes (ocho pisos ecológicos y más de 84 microclimas) que hacen que muchos insumos sean únicos en la región, lo que ha permitido su libre desarrollo y variedad, aunado todo esto también a las elaboraciones muy propias de cada zona, sumándole a ello la mixtura cultural (multirracial y pluricultural) que envuelve al país a través de su historia y sus movimientos migrantes.

Es por ello que, por ejemplo, el año 2013 se señaló en CNN que el tour gastronómico por Cusco y Lima es uno de los mejores del mundo:

"Nuestra comida se ha convertido en uno de los más grandes atractivos turísticos de nuestro país. Luego de que el Perú fuera elegido el mejor destino culinario del planeta en los World Travel Awards, un recorrido específico es el que hoy resalta. El portal de noticias de CNN eligió a un paseo por Lima y Cusco, organizado por la empresa Epitourean, como uno de los mejores tours gastronómicos exóticos del mundo" (Fuente 2013).

Estas consideraciones otorgadas al país y su particular gastronomía han generado en los turistas que llegan un aumento en sus expectativas sobre lo que van a encontrar, además del aspecto histórico-cultural que siempre nos ha caracterizado.

El problema que se observó radica en que a pesar de existir un tipo de turista muy ávido del servicio de $A \& B$, este no encuentra o no se satisface con la oferta dada, a pesar de que la demanda está presente y en auge. Haciendo números y tomando los datos del 2013, teniendo como referente que el $77 \%$ de los turistas que llegan a Lima visitan el centro histórico, se puede contabilizar que 145,992 turistas extranjeros han estado fluctuando en dicha zona a lo largo de un mes, para lo cual 4,866 extranjeros al día recorren sus veredas, sin tener en cuenta las temporadas de altas y bajas en el sector turismo.

A todo esto se haría bastante más difícil agregar al turista del interior del país que llega a diario al centro, de visita o por una gestión particular. Sumado a todo esto tenemos al limeño promedio que también fluctúa en la zona. Es decir, tenemos un flujo de visitantes muy alto que genera una demanda de la misma magnitud.

La propuesta se centra en que la oferta no satisface la demanda que se presenta, además que no hay un proyecto formal o que se haya difundido para desarrollar este aspecto del turismo que está creciendo cada vez más, para felicidad de la pequeña y mediana empresa dedicada a este rubro. En este caso, la Municipalidad de Lima es la llamada a cubrir tal deficiencia, ya que es la que tiene en primera instancia el acceso al catastro de todos y cada uno de los establecimientos de $A \& B$ que se ofertan en todo el centro histórico; luego viene el área de Defensa Civil y, en menor responsabilidad pero con parte de ella, el Ministerio de Salud, ya que se supone que sin su aprobación ningún establecimiento debería funcionar.

Revisando la poca bibliografía al respecto, es necesario mencionar el Plan Maestro del Centro Histórico de Lima al 2025, elaborado por la misma Municipalidad de Lima Metropolitana (2014) en la gestión anterior, en el cual -dentro de los objetivos estratégicos para Lima- se dice:

"Posicionamiento del centro histórico como motor de desarrollo turístico de la ciudad de Lima. El turismo constituye un elemento fundamental en la vida y en la economía de los centros históricos. En el umbral del siglo XXI, las ciudades históricas y los conjuntos patrimoniales se encuentran estrechamente asociados al turismo.

El turismo en la ciudad de Lima es básicamente de carácter cultural y gastronómico, representado por sus áreas urbanas históricas y la variedad de sus comidas.

De acuerdo a los planes de desarrollo del país, donde la potencialización del sector turístico alcanza uno de los primeros lugares en la economía, la propuesta para el centro histórico debe integrar 
los esfuerzos de restauración y rehabilitación bajo un enfoque de viabilidad económica dentro del cual la actividad turística juega un papel dinamizador y generador de recursos"

A pesar de los objetivos que plantea la municipalidad, no es fácil acceder a la información con exactitud; los servicios de A\&B alrededor de este espacio determinado no están actualizados; con menor rigurosidad se indica cómo están planteados, sin tener en cuenta otro elemento que se desarrolla también en el centro histórico: el comercio ambulatorio de alimentos, principalmente en horas de la noche, cuando hay poca o nula vigilancia sobre el tema y las condiciones en que se expenden ya corren por cuenta y riesgo de la persona.

Para definir el espacio a investigar, se ha delimitado que el turismo gastronómico de Lima metropolitana y su campo de acción se sitúa en la parte central de la ciudad, formando un cuadrante entre la Av, Colmena con Tacna, hasta el puente Santa Rosa, de ahí el borde del rio Rímac hasta el Jr. Paruro y luego hasta el cruce con la Av. La Colmena, en el cual se incluyen los principales centros de atracción turística y los servicios de $A \& B$ correctamente constituidos materia de investigación.

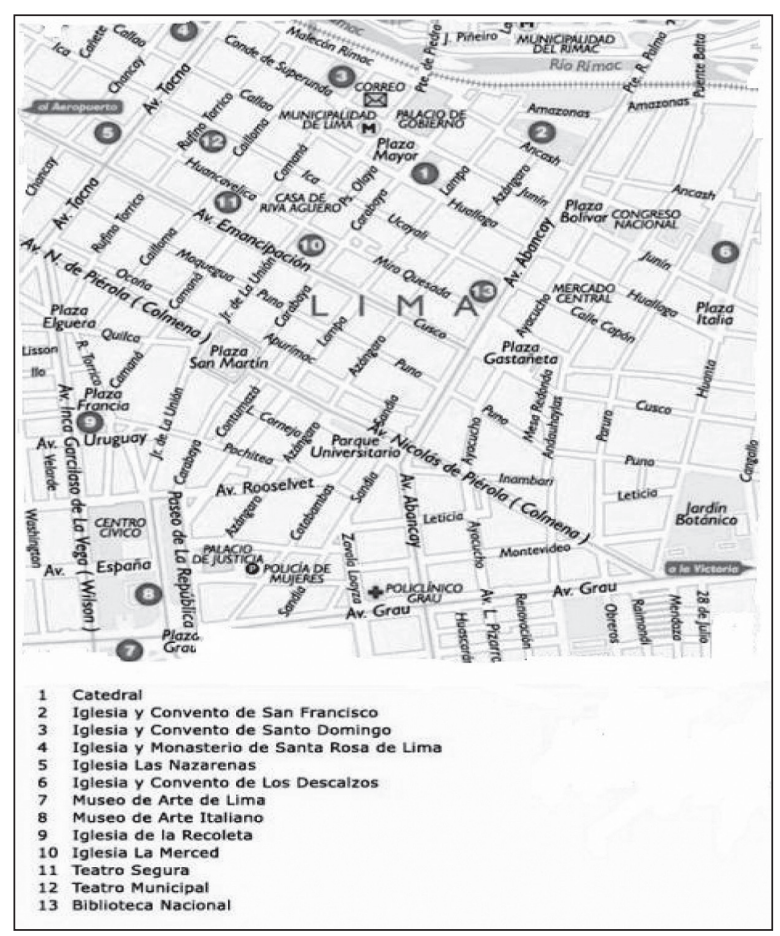

Fig. 1. Plano del Centro Histórico de Lima

\section{OBJETIVOS \\ Objetivo general}

Conocer las necesidades y expectativas de los turistas con relación a los servicios de alimentos y bebidas en el centro histórico, a su vez diagnosticar la oferta gastronómica que se brinda.

\section{Objetivos específicos}

- Analizar la oferta existente de un turismo gastronómico en Lima.

- Caracterizar el perfil del turista y determinar su comportamiento.

- Elaborar el diagnóstico y proponer alternativas para mejorar la oferta e incrementar la satisfacción del turista.

- Difundir el proyecto de investigación y los resultados a los actores involucrados, Asociación Peruana de Gastronomía, Municipalidad de Lima, Ministerio de Salud.

\section{JUSTIFICACIÓN}

El impacto de la cocina peruana en los últimos años se ha visto reflejado en el crecimiento de los restaurantes y otros establecimientos vinculados a la gastronomía, lo que ha generado no solo una valorización de los productos peruanos, sino también flujo de turismo interno de una zona a otra de nuestro país. Asimismo, la gastronomía se viene consolidando como uno de los ejes fundamentales de la política nacional de turismo, por lo que el Ministerio de Comercio Exterior y Turismo ha venido desarrollando el plan de política cultural del Perú en el exterior, que contempla la promoción de la gastronomía peruana, incidiendo en Lima como capital gastronómica. También cabe resaltar el Plan Nacional de Calidad Turística del Perú (CALTUR) (MINCETUR 2011), iniciativa gubernamental orientada a generar un movimiento hacia la excelencia mediante la combinación de servicios y facilidades que incremente la demanda turística.

De otro lado, el Perú ha sido elegido por segundo año consecutivo como el mejor destino culinario del mundo en los World Travel Award, el premio más prestigioso de la industria turística, habiendo participado junto a China, Francia, México, España, Italia, Japón, Malasia, Australia y Estados Unidos, lo que indudablemente genera expectativa y flujo turístico receptivo, más aún 
tomando en cuenta que se estima un $20 \%$ del gasto promedio per cápita en alimentación. Estas razones son fundamentales para identificar y analizar la oferta que brindamos a los turistas y asegurar su calidad respondiendo a las expectativas del turista y en beneficio del desarrollo de nuestro país.

\section{MARCO TEÓRICO}

De acuerdo a la Organización Mundial de Turismo (OMT) (2007), el turismo es un fenómeno social, cultural y económico relacionado con el movimiento de las personas a lugares que se encuentran fuera de su lugar de residencia habitual por motivos personales, de negocios o profesionales. Estas personas se denominan visitantes (que pueden ser turistas o excursionistas; residentes o no residentes) y el turismo tiene que ver con sus actividades, de las cuales algunas implican un gasto turístico. A su vez se define la gastronomía como la conjunción de aspectos culinarios con aspectos culturales que hacen a cada sociedad o comunidad. Por ello, la gastronomía no es simplemente un conjunto de técnicas o métodos de cocción, sino también la relación que los individuos establecen con el medio que los rodea, del cual obtienen sus recursos alimenticios, el modo en que los utilizan y todos aquellos fenómenos sociales o culturales que tienen que ver con la consumición de las preparaciones culinarias.

Hoy en día la gastronomía peruana goza de una reputación muy reconocida por propios y extraños; diversas empresas dedicadas al rubro de la restauración y el turismo valoran de manera sostenida sus bondades a partir de sus características culinarias como producto de años de fusión entre la cultura autóctona de las regiones y el aporte de los diversos grupos migrantes que llegaron y se desenvolvieron en estas tierras. La gastronomía peruana es la clara muestra de esa simbiosis cultural y racial que caracteriza al Perú.

De esta manera, esas mismas particularidades hacen que su atractivo y expectativa al respecto crezcan en el turista, es decir, se consolide un aspecto más para invertir su dinero esperando recibir a cambio un buen servicio al respecto. $\mathrm{Si}$ bien es cierto todos requieren de alimentación y su demanda se genera de manera natural, esta nueva opción bien llevada y marketeada hacia el turista significa una opción de desarrollo para las diversas empresas dedicadas al rubro.
Estudios concretos sobre lo anteriormente señalado entre turismo, gastronomía y centro histórico no hay; solo artículos periodísticos en revistas de circulación (como Somos, del diario El Comercio, por citar una fuente confiable), dan a conocer las noticias al respecto. Asimismo, la propia municipalidad cuenta con un registro de permisos o licencias para el funcionamiento de locales destinados a este rubro; pero como sucede en el país, muchas veces un permiso para desarrollar un servicio pasa con el tiempo a usarse para otro distinto; haciendo con ello que la información que brinda la municipalidad no siempre sea la correcta o, en muchos de los casos, esté simplemente desactualizada.

Otro referente importante para ubicar y conocer los servicios de A\&B y el estado de conservación en el Centro Histórico debe ser Defensa Civil, que en muchos casos está adscrito a la misma municipalidad y en otros, la institución misma -de manera independiente- se encarga de las visitas y permisos de funcionamiento. Cabe aseverar que si un local, no cuenta con la autorización de Defensa Civil pues no puede funcionar. En este caso, los registros están incompletos o simplemente según el catastro que se viene desarrollando, muchos locales no cuentan con dicho permiso y por ello no están registrados, así como también los permisos han caducado o se han hecho modificaciones que no se han notificado.

Un tercer referente válido para conocer la ubicación del local es el permiso de funcionamiento expedido por el la municipalidad para el local (que incluye obligaciones como las fumigaciones periódicas, por ejemplo). Para saber el número de trabajadores y su estado de salud se puede averiguar en centro de salud, tales como hospitales o dependencias afines del mismo municipio metropolitano, ya que los trabajadores deben contar con carnet de salud al día y que solo tiene validez por seis meses; para lo cual también se requiere que miembros del área hagan las visitas respectivas.

Expuesto todo esto, se puede afirmar que la información al respecto y el cruce de ella no existe de manera oportuna o fluida, ya que muchas veces cada área está por separado y son los mismos dueños o representantes que, de manera responsable, tienen que hacer cada trámite por separado para poder ofrecer su servicio de manera legal y ordenada. Trabajos al respecto no hay, por ello la propuesta busca ser un punto de partida y reflexión que analice dicho problema. 


\section{HIPÓTESIS}

De acuerdo a lo señalado y analizando los primeros resultados de la investigación, estos coinciden en que la mayoría de servicios de $\mathrm{A} \& \mathrm{~B}$, en relación con lo que el turista del centro histórico requiere, no satisfacen sus necesidades porque, por ejemplo, carecen de personal capacitado para brindar un servicio de calidad. Dicha capacitación va desde su poca o nula preparación hasta la falta del conocimiento básico del idioma inglés. Estos son algunos de los factores que desvirtúan nuestra imagen como destino gastronómico; por ello, la hipótesis de trabajo es:

"La capacitación de las empresas de gastronomía que constituyen la oferta para el turismo gastronómico contribuye a la satisfacción del turista y al desarrollo del turismo".

\section{MÉTODOS}

El tipo de investigación a desarrollar en la propuesta es de carácter descriptivo. Para ello se ha determinado acopiar información de campo a partir del levantamiento de un catastro referente al tema de la investigación, el cual está permitiendo reconocer con mayor detalle la real situación de los servicios de alimentos y bebidas de la zona del centro histórico; así como las características de identificación y sus necesidades. Esta información será contrastada con el comportamiento del turista y sus exigencias frente a dicha oferta, con la finalidad de elaborar un diagnóstico de la situación y plantear alternativas para su desarrollo y mayor satisfacción del turista.

Las fuentes de información requeridas y que se conjugan con el catastro vienen siendo elaboradas acorde a las necesidades de la propuesta, y son:

- Observación directa.

- Visitas a diversos establecimientos.

- Entrevistas.

- Cuestionarios.

- Indicadores estadísticos.

- Documentos oficiales ya existentes (municipalidad, Cámara de Comercio).

\section{RESULTADOS}

De acuerdo a los primeros resultados producto del trabajo de campo de indagación de la información y cubriendo ya el perímetro seleccionado del centro histórico, tenemos lo siguiente:

* La Municipalidad de Lima Metropolitana no posee un registro actualizado de todos los servicios de A\&B que se brindan en la zona, más aún en el horario de la noche donde el comercio ambulatorio es notorio.

* No hay controles periódicos ni coordinados entre la Municipalidad de Lima metropolitana, Defensa Civil o el Ministerio de Salud acerca de las condiciones en las cuales funcionan los servicios de $A \& B$ en la zona.

* Existe una total confusión entre los aspectos de responsabilidad con respecto a los servicios de $A \& B$ entre lo que le compete a la municipalidad y al Ministerio de Salud.

* Las revisiones que debería hacer Defensa Civil con respecto a estos servicios no se llevan a cabo.

* En muchos casos a simple vista se nota que las instalaciones no brindan las condiciones mínimas para su funcionamiento.

* El personal que está a cargo del servicio al cliente, en su mayoría, no está capacitado para cumplir tal labor.

* No hay una exigencia en el uso de uniformes acordes con la manipulación de alimentos por parte de los trabajadores que los preparan directamente.

* En muchos casos se niegan a presentar el carné de salud, elemento fundamental para quienes trabajan en el área de A\&B.

Dentro de los resultados preliminares más saltantes y de fácil detección están los ya expuestos, pero cabe resaltar que existen también empresas legalmente constituidas que trabajan dentro de las reglas estipuladas y, por su carácter de franquicia, cuentan con protocolos estándares en todas sus áreas; es por ello que el turista muchas veces las prefiere en primera instancia, siendo para ellos una marca conocida y de confianza.

Podemos señalar la presencia de Tanta en el centro histórico, reconocida cadena de origen nacional y con presencia en otros países de la región. 


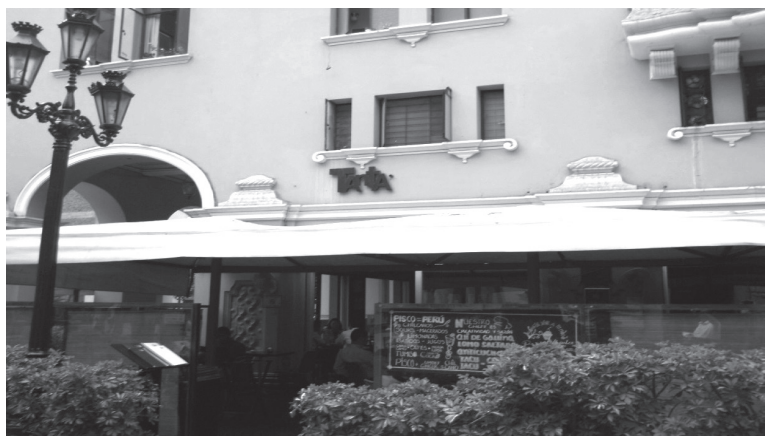

Fig. 2. Tanta

es una franquicia de nivel internacional que se encuentra presente en Estados Unidos, Perú, Chile, España, Colombia $y$, desde el año 2009, en Bolivia.

La pollería Pardos Chicken está presente en el centro histórico con una considerable demanda por su servicio al nivel del turista tanto internacional como nacional.

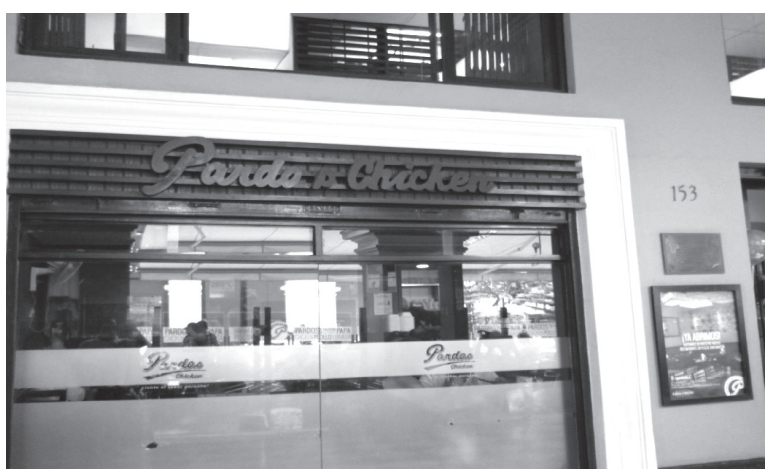

Fig. 3. Pardo's Chicken

Franquicia peruana de restaurantes y pollerías, especializada en pollos a la brasa y parrillas.

También se puede apreciar la presencia de diversos servicios de franquicias transnacionales. Para citar solo algunos nombres, están McDonald's o Papa John's, por ejemplo.

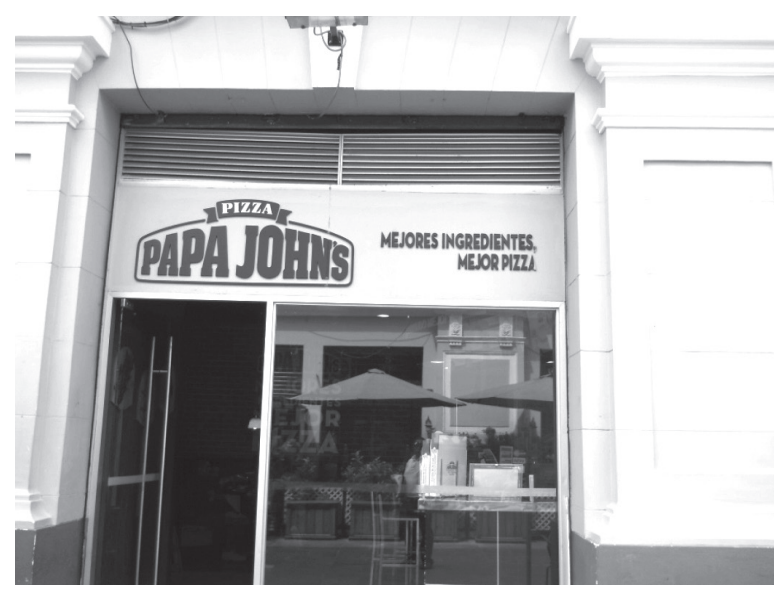

Fig. 4. Papa John's

Pizza - Los expertos en la preparación de pizzas de la mejor calidad.

\section{DISCUSIÓN}

La propuesta describe un problema que no es ajeno a nadie y está plasmado de alguna manera en la idiosincrasia nacional: la informalidad, o sacarle el mejor provecho a alguna situación y que esta no represente ningún atisbo de inversión, a la que muchos le asignan el calificativo de pérdida. El rubro de $A \& B$ requiere, por sobre todo, un alto grado de responsabilidad con la sociedad. Los alimentos son elementos muy delicados; por ello, la cadena de trabajo debe ser ordenada y claramente organizada, desde la recepción y las diversas formas de almacenamiento, según la naturaleza de cada insumo. Las temperaturas son fundamentales para la conservación de carnes, pescados y mariscos, por ejemplo. La correcta manipulación permite obtener un servicio de calidad y que sea eficiente. Mejorar las condiciones del local, desde las instalaciones eléctricas, pasando por las sanitarias, la ubicación de gas, los ductos de ventilación, las campanas extractoras, las mesas, lencería; vajilla, cubertería y cristalería (si fuese necesario), aunado a ello el tema de la capacitación del personal, hacen posible constituir un servicio de calidad.

En el Centro Histórico, establecer una categorización aleatoria que permita explicar la situación del servicio de $A \& B$ que relacione la calidad en el servicio y el nivel de formalidad es indirectamente proporcional; los locales de servicio de A\&B que cumplen con las normas constituyen un número muy limitado frente al universo de oferta gastronómica. A la vez, al otro lado de la muestra la mayoría de los servicios visitados no cumplen con los estándares de calidad para brindar un servicio acorde a los requerimientos del turista. Es en este nivel que se justifica la propuesta, ya que otro índice a tener en cuenta es que el turista internacional en su mayoría no consume necesariamente lo que el centro histórico oferta.

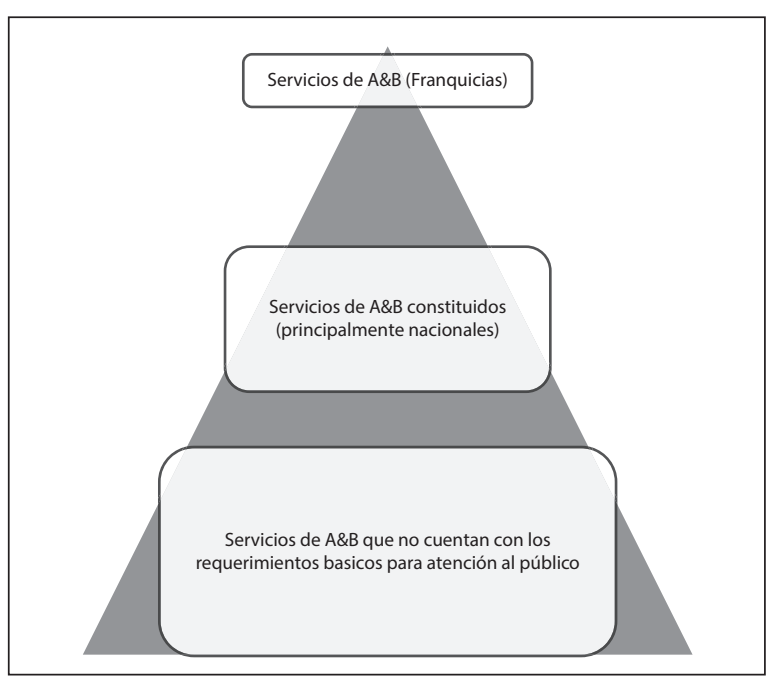




\section{CONCLUSIONES}

1. De acuerdo con lo observado en las primeras etapas de la investigación, se hace necesario establecer vía la Municipalidad de Lima metropolitana un censo de todos los servicios de A\&B no solo alrededor del Centro Histórico, sino también del área que comprende el Cercado de Lima, dadas las paupérrimas condiciones en que en muchas casonas y solares se expenden los alimentos.

2. La elaboración de la presente propuesta no es solo un tema que atañe al sector turismo, sino que también se puede apreciar que involucra a toda la sociedad que fluctúa y consume en la zona, constituyéndose un problema de salud ciudadana, incluyendo a los niños y ancianos residentes.

3. Es necesario establecer cadenas de responsabilidad con respecto a este tema, empezando con la propia municipalidad y los demás sectores involucrados.

4. Las empresas correctamente constituidas y debidamente capacitadas se volverán atractivas para el turista, sea este internacional o nacional. La confianza del cliente en el servicio de $A \& B$ que se le brinda es fundamental para su desarrollo como emprendimiento, así como para cada uno de sus trabajadores.

\section{LITERATURA CITADA}

“¿Cuál es el perfil del turista que visita Lima?”. (2014). En: Diario El Comercio. Fecha de consulta: 23/07/2015. Disponible en: <http:// elcomercio.pe/vamos/noticias/cual-perfilturista-que-visita-lima-noticia-1759010> .

"CCL: Turismo receptivo en el Perú crecería 6\% en 2015”. (2015). En: Peru21. 29 de enero del 2015. Fecha de consulta: 29/01/2015. Disponible en: < http://peru21. pe/economia/ccl-turismo-receptivo-creceria-6-2015-2210593>.
Arellano Marketing. (2009). "El aporte económico y social de la gastronomía en el Perú”. En: Instituto del Perú. (s.f.). USMP, Lima. Fecha de consulta: 29/01/2015. Disponible en: < http://www.institutodelperu.org.pe/ descargas/Eventos/Conferencias/2009sepmicroseguros/aporte_de_la_gastronoma_en_el_per.pdf > .

Comisión de Promoción del Perú para la Exportación y el Turismo (PROMPERÚ). (2014). Perfil del turista extranjero 2013. PROMPERÚ, Lima. Fecha de consulta: 05/01/2015. Disponible en: < http://media.peru.info/ IMPP/2013/TurismoReceptivo/DemandaActual/PerfilTuristaExtranjero2013.pdf>.

"Tour gastronómico por Cusco y Lima es uno de los mejores del mundo, según CNN" (2013). En diario El Comercio. Fecha de consulta 05/01/2015. Disponible en: <http:/ elcomercio.pe/gastronomia/peruana/tourgastronomico-cusco-lima-uno-mejoresmundo-segun-cnn-noticia-1528011>.

Ministerio de Comercio Exterior y Turismo (MINCETUR). (2011). "Plan Nacional de Calidad Turística del Perú (CALTUR)”. En: MINCETUR. Fecha de consulta: 05/01/2015. Disponible en: <http://www.mincetur.gob. pe/Turismo/Otros/caltur/pdfs_documentos_ Caltur/Plan_CALTUR_actualizado.pdf $>$.

Municipalidad de Lima Metropolitana. (2014). Plan Maestro del Centro Histórico de Lima al 2025. PROLIMA, Lima. Fecha de consulta: 12/02/2015. Disponible en: < http://www. munlima.gob.pe/imagenes/descargas/programas/prolima/PLANMAESTRO.pdf $>$.

Organización Mundial de Turismo (OMT). (2007). Entender el turismo: Glosario básico. World Tourism Organization Network, Madrid. Fecha de consulta: 12/01/2015. Disponible en: < http://media.unwto.org/es/content/ entender-el-turismo-glosario-basico $>$. 